\title{
The Placebo Effect in Sport: How Practitioners Can Inject Words to Improve Performance
}

Placebo effects involve a complex interplay between an intervention and factors associated with the administration of that intervention, such as expectations, previous experiences, and interactions between participant and researcher. ${ }^{1}$ (We refer to intervention as any interference aimed at improving or maintaining an athlete's performance, such as pharmacological/nutritional [eg, caffeine], psychological [eg, imagery], physical [eg, massage], or mechanical [eg, altitude training].) While researchers often regard the placebo effect as a nuisance to control for in randomized control trials, for practitioners (eg, sport scientists, coaches, physiotherapists), it can be a powerful tool to augment the beneficial effects of an intervention.

In the last 2 decades, a growing body of research has identified various neurobiological mechanisms of the placebo effect, with a significant number of studies investigating the mediating role of the opioid, endocannabinoid, serotonin, and dopamine systems. ${ }^{2}$ In sport and exercise science, understanding the full range of placebo effects and the underpinning mechanisms is important in how they are likely to influence the effectiveness of interventions athletes use (eg, caffeine, cold-water immersion, altitude training). ${ }^{3}$ Of the few systematic review papers in this area, we reported that the placebo effect has a small to moderate effect on nutritional and mechanical interventions, with larger effects shown for banned interventions, such as anabolic steroids and erythropoietin, and when participants had prior experience of using the intervention. ${ }^{4}$

Research on the placebo effect indicates that words about the effectiveness of an intervention significantly affect the outcome on performance. For example, after ingesting caffeine, athletes run faster when they are told it is caffeine than when they are told it is a placebo. ${ }^{5}$ Similarly, when athletes ingest a placebo, they run faster when they are told it will improve performance than when they are told that it will worsen performance. ${ }^{6}$ If athletes are told that they received an intervention that significantly improves performance, they are more likely to exhibit greater improvements in performance than if they are told it is benign. ${ }^{7,8}$ For researchers using randomized controlled trials, it is, therefore, important that communication be standardized between conditions and participants to control for placebo effects that may underestimate or overestimate the efficacy of an intervention.

The placebo effect is underpinned by beliefs that an intervention will or will not exert an effect. Athletes are often administered numerous interventions to support various aspects relating to recovery, injury, and peak performance (eg, cold-water immersion, altitude training, and protein supplements). Sport practitioners should be cognizant of the words they use when administering these interventions to develop their athletes' belief that the intervention is effective. Consider, for example, the case of a physiologist introducing altitude training into an athlete's training program. The physiologist might provide the following disclosure to their athlete:

I recommend that we include altitude training in your program. Several studies have shown that altitude training can be effective for increasing the volume of red blood cells, allowing your cardiovascular system to carry more oxygen, improving endurance performance and muscle-buffering capacity. Collectively, the use of altitude training is very beneficial for your training and performance.

This disclosure is honest, based on evidence, and aimed at engendering a positive belief in the effectiveness of altitude training. The physiologist applies an understanding of altitude training and the placebo effect through words to potentiate the response.

At the 2017 Inaugural International Symposium on Placebo Effects in Sport and Exercise, Professor Fabrizio Benedetti stated that, to elicit changes in people's physiology, we do not necessarily inject drugs but words. While Benedetti was referring to the methods used by placebo-effect researchers, this statement holds true for all practitioners working with athletes. Practitioners do not work with their athletes in a vacuum, but in a complex environment of physiological and psychological states that vary from athlete to athlete and from situation to situation. Almost every aspect of the administration of an intervention moderates the placebo effect. It is, therefore, important that practitioners recognize that the placebo effect can significantly affect the outcome of an intervention. Through an injection of words about the effectiveness of an intervention, practitioners can use knowledge and understanding of the placebo effect to maximize the likelihood of improvements in performance.

Bart Roelands, IJSPP Associate Editor,
Vrije Universiteit Brussel, Belgium,
and Canterbury Christ Church University,
United Kingdom
Philip Hurst,

\section{References}

1. Beedie C, Benedetti F, Barbiani D, et al. Consensus statement on placebo effects in sports and exercise: the need for conceptual clarity, methodological rigour, and the elucidation of neurobiological mechanisms. Eur J Sport Sci. 2018;18(10):1383-1389. doi:10.1080/ 17461391.2018.1496144

2. Colagiuri B, Schenk LA, Kessler MD, Dorsey SG, Colloca L. The placebo effect: from concepts to genes. Neuroscience. 2015; 307:171-190. PubMed ID: 26272535 doi:10.1016/j.neuroscience. 2015.08.017

3. Rohsenow DJ, Marlatt GA. The balanced placebo design: methodological considerations. Addict Behav. 1981;6(2):107-122. PubMed ID: 7023202 doi:10.1016/0306-4603(81)90003-4

4. Hurst P, Schiphof-Godart L, Szabo A, et al. The placebo and nocebo effect on sports performance: a systematic review [published 
online ahead of print August 20, 2019]. Eur J Sport Sci. 1-14. doi:10.1080/17461391.2019.1655098

5. Hurst P, Schiphof-Godart L, Hettinga F, Roelands B, Beedie C. Improved 1000-m running performance and pacing strategy with caffeine and placebo: a balanced placebo design study. Int J Sports Physiol Perform. 2020;15(4):483-488. doi:10.1123/ijspp.20190230

6. Hurst P, Foad AJ, Coleman DA, Beedie C. Athletes intending to use sports supplements are more likely to respond to a placebo. Med Sci
Sports Exerc. 2017;49(9):1877-1883. doi:10.1249/MSS.0000000 000001297

7. McClung M, Collins D. "Because I know it will!": placebo effects of an ergogenic aid on athletic performance. J Sport Exerc Psychol. 2007; 29(3):382-394. PubMed ID: 17876973 doi:10.1123/jsep.29.3.382

8. Saunders B, de Oliveira LF, da Silva RP, et al. Placebo in sports nutrition: a proof-of-principle study involving caffeine supplementation. Scand J Med Sci Sports. 2017;27(11):1240-1247. PubMed ID: 27882605 doi: $10.1111 /$ sms. 12793 